\title{
Metodología para la enseñanza de las Ciencias Naturales empleada por docentes costarricenses de las escuelas Vesta, Jabuy y Gavilán pertenecientes a la comunidad indígena Cabécar
}

\author{
Vílchez-Durán, Cynthia \\ Metodología para la enseñanza de las Ciencias Naturales empleada por docentes costarricenses de las escuelas \\ Vesta, Jabuy y Gavilán pertenecientes a la comunidad indígena Cabécar \\ Revista Educación, vol. 43, núm. 1, 2019 \\ Universidad de Costa Rica, Costa Rica \\ Disponible en: http://www.redalyc.org/articulo.oa?id=44057415038 \\ DOI: https://doi.org/10.15517/revedu.v43i1.27673
}

Esta obra está bajo una Licencia Creative Commons Atribución-NoComercial-SinDerivar 3.0 Internacional. 


\section{Metodología para la enseñanza de las Ciencias Naturales empleada por docentes} costarricenses de las escuelas Vesta, Jabuy y Gavilán pertenecientes a la comunidad indígena Cabécar

Methodology for Teaching Natural Sciences Used by Costa Rican Teachers at the Vesta, Jabuy and Gavilan Schools in the Cabécar Indigenous Community

Cynthia Vilchez-Durán

Universidad Estatal a Distancia, Costa Rica

DOI: https://doi.org/10.15517/revedu.v43i1.27673

cvilchez@uned.ac.cr

(iD http://orcid.org/0000-0002-6591-4554

Recepción: 12 Enero 2017

Aprobación: 04 Febrero 2019

\section{RESUMEN:}

El presente artículo analiza las estrategias didácticas empleadas por el personal docente de tres escuelas pertenecientes a la comunidad indígena Cabécar en la enseñanza de las Ciencias Naturales a partir de una investigación sustentada metodológicamente en el paradigma cualitativo. Para la obtención de la muestra se utilizó el método no estadístico, seleccionando las escuelas Vesta, Gavilán y Jabuy, las cuales presentaban características aptas en cuanto a su acceso y condiciones climáticas. La recolección de datos se realizó mediante observaciones de las estrategias didácticas utilizadas por el cuerpo docente en el aula, además de entrevistas dirigidas al estudiantado. Una vez transcritos los resultados se realizó una contrastación de la información determinando las estrategias predominantes. Entre los principales hallazgos del estudio se evidencia el uso de estrategias con enfoque conductista, orientadas a una práctica de enseñanza tradicional-expositiva mientras que, por otro lado, el alumnado demanda estrategias que incluyan actividades con más recursos educativos, juegos y exploraciones fuera del aula. En conclusión, las estrategias didácticas se caracterizan por el papel protagónico activo del profesorado, relegando al estudiantado a una posición de receptor pasivo, quienes por el contrario emplazan clases de ciencias más activas, en donde la pizarra no se convierta en el único instrumento de enseñanza.

Palabras ClaVE: Indígena, Cabécar, Ciencias, Educación, Estrategias.

\section{Abstract:}

This article analyzes strategies for teaching the Natural Sciences used at three schools in the Cabécar Indigenous community. The research methodology is based on a qualitative paradigm. The sample group was obtained through a non-statistical method and selected from the Vesta, Gavilán and Jabuy schools since these schools could by easily accessed given the good weather conditions in the area. Data was collected from observing teaching strategies in the classroom and student interviews. After recording the results, we compared the information to determine the most predominant strategies. The main findings included strategies used for enforcing good conduct geared towards traditional expository teaching. But, overall, students now expect strategies that require more educational resources, such as games and exploratory field trips outside of the classroom. To conclude, although teaching strategies are characterized by the active role of the teacher as the protagonist with students as passive recipients, students tend to demand more active science classes, for example, where the blackboard is not the only teaching aid.

KEYwORDS: Indigenous communities, Cabécar, Sciences, Education, Teaching Strategies.

\section{INTRODUCCIÓN}

Para Rodríguez (2009), el desarrollo de la educación en las comunidades indígenas ha sido un proceso lento. Los primeros pasos se dieron mediante la emisión de algunos decretos desde la administración de Otilio Ulate Blanco en 1950; posteriormente algunas leyes promulgadas en el año 1973 realizaron las primeras alusiones a la educación indígena y es a través de instituciones como la Universidad de Costa Rica en donde se comenzaron a elaborar propuestas sobre el desarrollo de la educación para esta población. 
En materia propia de legislación dos acciones se identifican como las más relevantes; la primera se genera con la aprobación de la ley $\mathrm{N}^{\circ} 7316$ Convenio sobre los Pueblos Indígenas y Tribales en Países Independientes, promulgada en noviembre de 1992, la cual se basa en el Convenio 169 del año 1989 de la Organización Internacional del Trabajo (OIT), este tuvo como propósito principal establecer un instrumento internacional para exigir derechos y condiciones económicas, sociales y culturales para fortalecer la dignidad y atributos de la población indígena como seres humanos.

En el tema educativo dentro del citado Convenio 169 de la OIT, destacan los artículos No. 26, 27, 28 y 29 en los cuales para el diseño de políticas educativas se plantea considerar aspectos claves influyentes directos en el proceso educativo de las comunidades indígenas, tales como recursos, su lengua, sus tradiciones, entre otros.

En segunda instancia, el 25 de febrero de 1993 el gobierno en turno emite el Decreto Ejecutivo No 22072 MEP mediante el cual se creó el Subsistema de Educación Indígena, en el que se define como objetivo general "Desarrollar progresivamente la educación bilingüe y pluricultural en los territorios indígenas oficialmente reconocidos" (Estado de la Nación, 2012, p.318 ). La creación de ese Subsistema de Educación Indígena determina la necesidad de que el personal docente nombrado para los centros de educación de las zonas indígenas pertenezca a las mismas etnias.

Para el año 1994, se crea el Departamento de Educación Indígena del Ministerio de Educación Pública con la clara meta de mantener la adecuación curricular y la enseñanza de las lenguas maternas. Uno de sus principales logros es la institucionalización de la educación indígena para los pueblos indígenas.

De acuerdo con los datos del censo nacional realizado por el INEC (2011) en el año 2010, en Costa Rica la población indígena alcanza alrededor de 104.000 personas, lo cual representa un poco más de un $2 \%$ de la población nacional. Casi el 60\% de la población indígena habita en zonas rurales tales como el cantón de Talamanca, en Limón. Dentro de los grupos indígenas destaca la Cabécar, que habita los territorios de Chirripó Arriba, Chirripó Abajo, Nairí Awari, Tayni, Telire, Keköldi y Talamanca Cabécar.

Las comunidades indígenas se asientan sobre territorios rurales (de acceso restringido en algunos casos) con severas carencias en cuanto a medios de transporte, infraestructura vial, servicios públicos, medios de comunicación y recursos económicos. Por ejemplo, dentro del índice de desarrollo humano de los 82 cantones del país, Talamanca ocupa la posición No.81 superando solamente al cantón de Los Chiles. (Universidad de Costa Rica, 2016)

El acceso a la educación primaria en territorios indígenas alcanza un 66\% (INEC, 2011), sin embargo dicho porcentaje tiende a aumentar para las personas indígenas habitantes en la periferia de estos, así como para la población indígena ubicada en territorio no indígena, la asistencia a la educación básica aumenta hasta un 75\%, cifras en las que se reflejan cómo la accesibilidad depende mucho del espacio geográfico en el cual se encuentre el indígena. (INEC, 2011)

Lo anterior se ratifica con lo indicado por Solano (2000), residentes de los territorios indígenas se enfrentan a condiciones educativas desfavorables y estas tienden a mejorar un poco conforme se alejan de los centros de población; esto sugiere, en primera instancia la existencia de problemas de acceso al sistema educativo.

En la mayoría de las comunidades indígenas solamente existe el acceso a educación primaria y las posibilidades de poder continuar en la educación secundaria, implican abandonar sus comunidades y familias, La población en territorios indígenas alcanza tan solo el 9,1\% de asistencia a la educación secundaria, mientras quienes logran estudiar fuera de sus territorios obtienen un porcentaje superior de $33,2 \%$. INEC (2011)

Los territorios indígenas en promedio poseen un $37 \%$ de su población en el segmento de edad entre 0 y 14 años por lo que se torna importante analizar las oportunidades educativas que se les pueden brindar a personas menores y jóvenes a través de la enseñanza primaria.

En relación con este tema, en el periódico La Nación, Villegas (2012), en una consulta al director de Gestión y Calidad Educativa del Ministerio de Educación Pública, se transcribe lo siguiente: 
Félix Barrantes, director de Gestión y Calidad Educativa del Ministerio de Educación Pública (MEP), consideró que los centros educativos donde nadie ganó el ingreso al sistema de bachillerato posiblemente funcionan en un entorno complicado. "Algunas veces son comunidades muy pobres, de integración familiar no completa y tienen problemas internos de edificios", manifestó. (2012)

Los factores citados anteriormente evidencian que la aplicación de la educación indígena representa una realidad multifactorial; en este sentido el análisis de estrategias didácticas en el área de las Ciencias Naturales provee una fuente de información oportuna que pretende emancipar el sentido mismo de la educación, al buscar la inclusión y ante todo la equidad.

Dado lo anterior el presente artículo plantea evaluar las estrategias didáctico- metodológicas para la enseñanza de las Ciencias Naturales empleadas por el profesorado de las escuelas unidocentes de Vesta, Jabuy y Gavilán pertenecientes a la comunidad indígena Cabécar, contribuyendo a la búsqueda de posibles soluciones en el proceso permanente de mejora en la entrega de la docencia, responsabilidad inherente del profesional en cualquiera de los contextos en los cuales se desenvuelva adaptándose a los recursos del entorno.

\section{MARCo TEÓRICo}

La función pedagógica del docente indígena contempla, a priori, el uso de varias herramientas, con la única funcionalidad de alcanzar sus propósitos.

El deber de todo el personal docente, sea cual sea el contexto en el cual se desenvuelva, es poner en juego su creatividad, con la finalidad de generar en el estudiantado un aprendizaje significativo; es decir, que logren dominar aquellos contenidos conceptuales, procedimentales y actitudinales exigidos en el currículo escolar, para insertarse dentro de los procesos de desarrollo, cada vez más exigentes, de un mundo globalizado y también en procesos de construcción de la paz y la justicia, para su familia, comunidad y el país. Siendo el estudiantado el principal actor transformador de su entorno y el profesorado el mediador de este proceso.

Para Alonso y Gallardo (2014) los siguientes son elementos básicos para el perfil de un docente intercultural:

Conocimientos: lingüísticos e histórico sociales de la cultura de las comunidades. Actitudes: Disposición a la construcción de ideas o proyectos con personas que piensan diferente, respeto por lo diferente, escucha y apertura. Habilidades: para la investigación y el análisis crítico, para la aplicación, desarrollo y evaluación de metodologías didácticas, para el diálogo (p.23).

Dada la importancia del papel pedagógico docente, se hace una breve descripción de algunos conceptos básicos tales como, el proceso de enseñanza-aprendizaje, la estrategia didáctica como recurso pedagógico; los paradigmas educativos, así como otros elementos involucrados en el proceso metódico-didáctico de las Ciencias Naturales.

\section{Los procesos de enseñanza y aprendizaje}

Nicoletti (2003), explica que "según la Real Academia de la Lengua, la enseñanza es entendida como el sistema y método de dar instrucción de un conjunto de conocimientos, principios o ideas” (p. 16).

En este sentido el mismo autor hace referencia también a especialistas en Metodología Didáctica quienes "advierten que la enseñanza es entendida en lo que tiene de aplicación del conocimiento, principio o idea". (Nicoletti, 2003, p.17)

A partir de lo anterior se puede definir a la enseñanza como una vía de conocimiento entre el estudiante y el docente, en este proceso la asimilación de saberes dependerá del tipo de enseñanza utilizado por el docente dentro de su estrategia didáctica mediante la cual ampliará el horizonte para el educando y evitará delimitar su aprendizaje a ciertas formas de enseñar. 
Para Us (2009) dentro del proceso de aprendizaje se conjugan tres principios:

1. El estudiantado que aprende, dando lugar al protagonismo del alumnado en el acto de aprender.

2. Los contenidos escolares como conocimientos científicos y culturales con los cuales se pone en contacto al estudiantado con lo que se espera pueda aprender.

3. El papel del cuerpo docente colaborando con el alumnado a construir significados y atribuir sentido a lo aprendido.

Por lo tanto, la entrega de la docencia asegura una adecuada relación entre la actividad mental constructiva de cada estudiante brindando significancia social y cultural a los contenidos de aprendizaje.

\section{Elementos del proceso enseñanza-aprendizaje desde algunos paradigmas educativos.}

Dentro del paradigma funcionalista (Morrows y Torres, 2002) se asume la condición del escolar dependiente de la interacción social, pues la adquisición de nuevos roles dentro de la dinámica educativa trae consigo oportunidades y crea vías de cooperación con otros estudiantes y también de competencia entre estos, en cuanto a calificaciones sumativas se refiere.

Otro paradigma se conoce como el conductual, basado principalmente en la enseñanza programada tal como la propuso Cruz (1986 citado por Hernández 1998) "es el intento por lograr en el aula los mismos resultados de control conductual que se alcanzan en los laboratorios, usando los principios conductuales" (p.10). Bajo una corriente conductista, al alumnado se le otorga únicamente el rol de receptor pasivo; en la visión conductista se genera un panorama cerrado y rígido, en el cual el cuerpo docente asume un papel protagónico y ejerce el control del proceso de enseñanza y guía la temática educativa a la contestación de respuestas cerradas sin ningún tipo de criticidad o reflexión.

Cuando se menciona el paradigma complejo dentro del proceso educativo, a este se le considera de tipo holístico, por cuanto no supone el aislamiento de conocimientos, sino por el contrario estos forman un todo relacionándose unos con otros. Desde este paradigma se visualiza una educación integral e integrada. (Morin, 2004).

Para Hernández, (1998) se denomina la enseñanza indirecta aquella proveniente del paradigma constructivista. Esta se encuentra concebida en una contundente actividad, acompañada de la curiosidad del alumnado y dirigida a descubrir poco a poco los elementos de la realidad que le rodea, para que desde ahí se produzca la significancia, construida a partir del medio y de lo aprendido por cada estudiante.

La educación científica del siglo XXI ofrece muchas alternativas metodológicas al personal docente, para desarrollar un proceso de enseñanza y aprendizaje significativo, atractivo y motivador, el cual inspire curiosidad en el estudiante por conocer y entender los fenómenos cotidianos a su alrededor y encontrar soluciones a las problemáticas que el medio demanda.

No obstante, las prácticas metodológicas o estrategias didácticas no pueden generalizarse, en lo referente a su aplicación por parte del profesorado. La enseñanza de las Ciencias Naturales enfrenta a docentes y a estudiantes a un complejo proceso de enseñanza-aprendizaje, el cual se ve afectado por diversos elementos.

Merino (1998) puntualiza que la forma en cual se ha enseñado la ciencia "ha desencadenado en los y las estudiantes falta de interés en las actividades por la tendencia a la memorización y repetición de una 'ciencia única' o acabada y desvinculada de la vida cotidiana” (p.21). Esta problemática se ve también reflejada en el diagnóstico hecho por el MEP en el año 2010, el cual, tal y como señala Villegas (2012), presenta las dificultades en el nivel de secundaria del aprendizaje de las Ciencias Naturales: "los y las colegiales patinan para resolver problemas de matemáticas y ciencias y los expertos atribuyen ciertos factores como mala formación docente y el método de enseñanza" (p.4). 
En un entorno altamente competitivo, exigente de altas dosis de creatividad y liderazgo en los diferentes campos, el personal docente debe reinventarse y mejorar año con año su metodología de enseñanza; encontrar el estilo de aprendizaje apropiado para sus estudiantes, permite solventar o disminuir la problemática planteada, desde el conocimiento de otras alternativas que se encuentran al alcance del docente.

\section{Las herramientas pedagógicas y estrategias didácticas}

Las herramientas para lograr aprendizajes, pueden ser de dos tipos: pedagógicas y didácticas.

Para Us (2009), "la principal herramienta pedagógica es la interacción social desde: la escuela, la comunidad y cualquier otro acceso (...) debe dar lugar a situaciones significativas" (p.110).

Por otra parte, se conceptualiza la estrategia didáctica como "una secuenciación de actividades planificadas para conseguir un aprendizaje" (Ontoria, 1999, p.36). En este aspecto se valora el trabajo previo docente visualizando y determinando cuáles experiencias didácticas le serán de utilidad de acuerdo con la contextualización del entorno a su alrededor, aunado a los objetivos por alcanzar dentro de la dinámica educativa.

Para Ontoria (1999), las principales estrategias didácticas, se pueden dividir en estrategias didácticas grupales, incluyendo foros, mesas redondas, paneles de trabajo, talleres, juegos didácticos, entre otras; sin seguir un planeamiento complejo, el docente puede adecuarlas al contexto que necesita. En contraparte se encuentran las estrategias didácticas individuales, las cuales proveen actividades con diferentes metodologías propiciando el protagonismo activo del educando. Las condiciones de estas estrategias versan en conocer las necesidades individuales de cada estudiante y adecuar la estrategia con su particularidad.

\section{Generalidades de la enseñanza de Ciencias Naturales para la escuela del siglo XXI}

\section{Didáctica de las Ciencias Naturales}

Liguori y Noste (2007) visualizan a las Ciencias Naturales como un campo científico en formación, el cual se constituye en un cuerpo coherente de conocimientos centrando su investigación en la problemática relacionada a la enseñanza y el aprendizaje de las ciencias, por cuanto los conocimientos científicos son específicos y por ello no se enseñan ni se aprenden como otros saberes (lingüísticos, matemáticos, etc.).

La actual concepción de ciencia ha generado un replanteamiento entendido por enseñar y aprender ciencias. Algunos aspectos destacados son el reconocimiento indiscutible del protagonismo del alumnado en el proceso de enseñanza- aprendizaje y la valoración de las interacciones producidas en el aula (docente/ estudiantes y estudiantes entre sí), como un factor fundamental en el logro de aprendizajes significativos Sanmartí (2002).

De la educación indígena a la educación científica

La definición de educación científica, se puede concebir como

un enfoque pedagógico orientado a formar ciudadanos capacitados para comprender, manejarse y participar en un mundo en el que la ciencia y la tecnología están cada día más presentes. Este enfoque ha tomado el nombre de Ciencia, Tecnología y Sociedad (CTS), el cual es especialmente apropiado para fomentar una educación tecno científica dirigida al aprendizaje de la participación, aportando un nuevo significado a conceptos tan aceptados como alfabetización tecno científica, ciencia para todos o difusión de la cultura científica (Gordillo y Osorio, 2003, p.23).

Parafraseando lo indicado por Valladares (2010) en cuanto a los obstáculos del aprendizaje de las ciencias en la cultura indígena se mencionan aspectos de tipo: a) Conceptual, porque se concibe a la ciencia como impropia a su entorno; b) Pedagógica, se torna incomprensible desde el punto de vista holístico, no existe una relación entre los conocimientos previos y los recién adquiridos, circunscribiéndose a la memorización de conocimientos ajenos al entendimiento; c) Ideológica, se atañe a considerar el fracaso escolar en la enseñanza 
de las ciencias y al mismo tiempo poca demanda en educación científica superior por parte del estudiantado; d) Práctica, los recursos didácticos de carácter científico no son inclusivos desde el punto de vista de diversidad cultural.

\section{Educación científica en la educación indígena: perspectivas}

Perspectiva pluralista del conocimiento: Los conocimientos tradicionales si bien se califican como saberes, no son científicos porque no han surgido ni pertenecen a ninguna tradición que desde el punto de vista sociológico, histórico y epistemológico se reconozca como científica; pero no por ello, como intenta mostrar el trabajo de Winch (1987), son irracionales o ilegítimos, pues con estos saberes muchas comunidades tradicionales cumplen sus fines prácticos y resuelven muchos de sus problemas. (p.83).

Perspectiva sociocultural de la educación basada en el logro de desempeños competentes: Desde el punto de vista pedagógico, una educación científica intercultural parte de la consideración de la diversidad cultural como un hecho clave para lograr la conexión estrecha entre los intereses y finalidades que se consideran relevantes y legítimos para el aula de ciencias, con aquellos considerados así por las distintas comunidades. Sen, citado por (Valladares, 2010, p.83)

La generación de programas educativos debe ser consonante con las características de las poblaciones meta, al respecto (Alonso et ál., 2013) para el diseño de programas de estudio bajo un desarrollo curricular intercultural sugieren "construir un piso conceptual común que articule teorías y posibilidades de entendimiento en torno al enfoque intercultural y bilingüe” (p.64).

Plantean los mismos autores cómo tales programas deben recuperar como parte de la noción de aprendizaje tres momentos claves:

- El conocimiento de la diversidad cultural, la cual supone construir una disposición y apertura tanto cognitiva como ética.

- El reconocimiento, que implica aprender de la diferencia como una decisión propia y no como una imposición.

- La valoración, requiere que el estudiantado resignifique las diferencias e intente construir una visión renovada con miras a lo común desde una posición intercultural.

Educación indígena en Latinoamérica

Para Cuevas (2013), En los países latinoamericanos donde la población indígena representa a un importante sector de la sociedad, la educación oficial de los grupos étnicos minoritarios se lleva a cabo en la lengua materna de las comunidades indígenas. En países como Colombia

la educación se enmarca dentro de las propias comunidades étnico-lingüísticas que, a través de sus organizaciones indígenas y en coordinación con entes institucionales han propuesto y promovido el reconocimiento de una educación oficial que se enmarque dentro de los límites de una cultura propia y una nacional. (Cuevas, 2013, p.1)

Otros países como Venezuela, Perú y Bolivia han desarrollado iniciativas para implementar programas de educación intercultural bilingüe, sin embargo, a pesar de tales esfuerzos persisten vacíos relacionados a temas como el uso de materiales didácticos, la generalización de los currículos escolares e incluso los calendarios en tiempo de clases.

Sobre la Política Educativa Indígena en México, Bastiani-Gómez, Ruiz-Montoya, Estrada-Lugo, CruzSalazar y Aparicio-Quintanilla (2012) subrayan que "la educación intercultural puede contribuir a superar los problemas de rezago en el desarrollo social, los conflictos interétnicos y la valoración de la identidad cultural y lingüística" (p.9), no obstante, en la práctica real la aplicación de este tipo de modelos suele enfrentarse contra el desconocimiento de los intereses educativos de estas poblaciones.

Los mismos autores mencionan cómo tales políticas intentan que docentes "adquieran habilidades metodológicas y curriculares para que puedan elaborar contenidos, materiales y estrategias en lengua indígena" (Bastiani-Gómez et ál., 2012, p.21) lo cual se ve limitado por el hecho de que los materiales didácticos de actualización docente son generalizados por parte de la autoridad educativa, caso similar a 
la realidad costarricense en donde el profesorado de los centros educativos indígenas tiene como principal medio de actualización las capacitaciones del MEP, las cuales son de carácter genérico.

Desde muchas aristas se puede visualizar que la enseñanza de las Ciencias Naturales en contextos multiculturales no solo representa un reto a nivel cognitivo, actitudinal y metodológico, puesto que debe ser considerada la cultura en todo su esplendor, sino que ésta también debe ubicarse dentro del campo educativo sujeto a las demandas de la globalización. El punto de inflexión entre la educación indígena y la educación científica, se puede originar a partir de los aportes de cada una, para el logro de un objetivo holístico, por ejemplo, buscar por qué, cómo, cuándo y dónde, y las soluciones a los problemas de la cotidianidad. Todos necesitan de este conocimiento, independientemente del contexto en el cual se desenvuelvan, el medio necesita respuestas a tantas interrogantes aún sin resolver.

\section{Procedimiento metodológico}

El paradigma de la presente investigación se encuentra en el enfoque cualitativo, específicamente, hacia "el estudio de un fenómeno proveniente de observaciones de los hechos y del razonamiento, interpretación y análisis de la realidad” indígena (Hernández, Fernández y Baptista, 2010, p. 515).

El tipo de investigación está determinado por el diseño etnográfico, que de acuerdo con Patton (2002, citado por Hernández et ál., 2010) "pretende describir y analizar ideas, creencias, significados, conocimientos y prácticas de grupos, culturas y comunidades” escolares (p. 697).

En concordancia con el objetivo de la investigación se realizó una categorización de las variables más relevantes en relación con el objeto de estudio centrando la metodología en tres ejes:

1. Perfil sociodemográfico de las personas participantes (docentes y estudiantes)

2. Estrategias didáctico-metodológicas utilizadas por docentes

3. Opiniones y percepciones de estudiantes sobre tales estrategias.

Los sujetos de la investigación son personal docente y estudiantado de las escuelas Vesta, Jabuy y Gavilán pertenecientes a la comunidad indígena Cabécar y ubicadas en el circuito 07 de la Dirección Regional de Educación de Sulá (Limón); de ellas, las escuelas Vesta y Jabuy son multigrado, en la primera la docente atiende de cuarto a sexto grado y en Jabuy el docente imparte a todos los niveles. En la escuela Gavilán trabajan docentes por cada nivel, en esta investigación fue seleccionado el nivel de quinto grado.

Entre los criterios utilizados para la elección de participantes se consideró la experiencia del profesorado (los tres, al momento de realizada la investigación, tienen más de dos años en la entrega de la docencia) además de la ubicación geográfica de las instituciones para que las condiciones del tiempo atmosférico no afectaran el acceso a estas escuelas dado el riesgo de exceso de lluvias y las constantes crecidas de los ríos. En lo concerniente al estudiantado se usó una muestra del $20 \%$ por cuanto para este tipo de población una amplia cantidad de participantes podría conducir resultados redundantes además del factor tiempo.

Para la recolección de la información se utilizaron las técnicas de la observación y el sondeo de opinión; en primera instancia se realizaron observaciones de las clases impartidas por los tres docentes en sus respectivas escuelas y específicamente en sus aulas (una observación por cada docente); el personal fue informado de previo y la información se registró en una bitácora o diario manual.

De manera previa se realizó una guía de observación en la cual se plantearon los aspectos relevantes de acuerdo con los objetivos planteados para la investigación. Tales aspectos se detallan a continuación:

A) Ambiente físico:

- Descripción de las aulas y los recursos didácticos con los cuales cuenta cada docente dentro del aula. 
B) Estrategia docente:

- Estrategias y metodologías didácticas utilizadas por el personal docente para los diferentes contenidos temáticos.

- Técnicas de enseñanza aplicadas por el profesorado.

- Instrumentos de enseñanza empleados.

- Contenidos temáticos en los cuales se basan las clases.- Contenidos temáticos en los cuales se basan las clases.

- Estilo de comunicación de cada docente con sus estudiantes.

C) Participación:

- Formas de corrección de cada docente hacia sus estudiantes.

- Participación del alumnado en clases.

- Actitud del estudiantado durante las lecciones.

- Desarrollo de trabajos individuales o grupales.

- Métodos de resolución de conflictos.

Como segundo instrumento se realizó para el sondeo de opinión una entrevista estructurada dirigida al estudiantado, para indagar información relacionada con las estrategias didácticas empleadas por el personal docente. La cantidad de estudiantes que se entrevistó en las escuelas Vesta, Jabuy y Gavilán se delimitó a 11 y 12 respectivamente, con edades entre 8 y 12 años. La recolección de los datos se efectuó de forma manual; la información recabada fue descrita textualmente de acuerdo con las expresiones de cada participante.

Para obtener la información del estudiantado el instrumento se elaboró en dos partes. La primera con los datos personales mientras que la segunda se les preguntó acerca de las estrategias empleadas enfocando las consultas sobre cuatro ejes:

1. Indagar si existe agrado por la clase impartida por el cuerpo docente.

2. Describir la forma por medio de la cual cada docente explica los temas.

3. Conocer los recursos utilizados por el personal docente.

4. Averiguar sobre la opinión del alumnado sobre el uso de otros insumos.

5. Por último, se les solicitó realizar un dibujo de lo que ellos entienden por ciencias, para esta actividad se les facilitó una hoja blanca de papel.

Previo a la investigación, la entrevista original fue sometida a la valoración de dos profesionales de la docencia en el campo de las Ciencias Naturales, ambos con experiencia en revisión de trabajos de investigación, los cuales externaron sus criterios ofreciendo algunos de mejora en cuanto al contenido de la entrevista, tales como:

- Desarrollo de una entrevista corta y redacción sencilla logrando preguntas más específicas.

- Cambio del enfoque de algunas preguntas con el fin de ubicar de mejor manera a la persona entrevistada, dentro del contexto solicitado y los objetivos requeridos.

- Eliminación de preguntas que se consideraban generadoras de respuestas redundantes.

Con los datos recolectados se procedió a contrastar dicha información con la teoría enmarcada en el presente estudio y de esta manera determinar las estrategias didácticas empleadas por el personal docente de escuelas pertenecientes a la comunidad indígena Cabécar para su respectivo análisis. 


\section{ANÁLISIS Y DISCUSIÓN DE RESULTADOS}

\section{Proceso de enseñanza ejercido por los y las docentes visto a partir de las observaciones realizadas}

\section{Recursos físicos y pedagógicos}

En relación con los recursos pedagógicos en el aula, las escuelas Vesta y Jabuy poseen pizarras de madera mientras en la escuela Gavilán se cuenta con una pizarra acrílica. Los centros educativos tienen mobiliario con marcadas diferencias, en las escuelas Vesta y Jabuy los pupitres son de madera y hierro, y denotan un deterioro generado por el uso durante los años, mientras tanto, en la escuela Gavilán los pupitres son de plástico especial y hierro en buenas condiciones, estos fueron donados por una empresa comercial.

Por otra parte, se denota una escasez de libros los cuales deben ser compartidos por el estudiantado mientras que ninguno de los centros educativos cuenta con recursos tecnológicos en el aula.

Proceso de enseñanza aprendizaje ejercido por el personal docente

En lo que respecta al proceso de enseñanza-aprendizaje, en la escuela Vesta la docente imparte lecciones de forma magistral para estudiantes de quinto grado, mientras tanto el alumnado de sexto grado realiza una práctica del libro de manera grupal acerca del tema ciencia y tecnología. La docente explica la importancia de la ciencia y la tecnología en la cotidianidad y cita ejemplos de artefactos útiles para el ser humano, tales como la cocina, la lavadora, el televisor, entre otros. Posteriormente, la docente le solicita al estudiantado realizar una práctica. En este punto cabe destacar que la docente no es de origen indígena, pero en la escuela asisten en su mayoría estudiantes indígenas.

En la escuela Gavilán, el docente utilizó la pizarra para explicar el tema mezclas homogéneas y heterogéneas enfocándose estrictamente en los contenidos relacionados con el tema. Con el objetivo de diferenciar entre una mezcla homogénea y heterogénea, formó pequeños grupos y repartió diferentes materiales, tales como arena, leche en polvo, agua, piedras, y dirigía paso a paso las actividades de sus estudiantes; además usó preguntas cerradas sobre los fenómenos tales como: ¿Se mezcla bien el agua y la arena? ¿La leche en polvo en agua se mezcla?

Por su parte, en la escuela Jabuy el docente explicó el tema fases de la luna utilizando para ello esquemas y dibujos realizados en la pizarra. Una vez terminada la explicación, les facilitó a sus estudiantes arcilla para la representación de las fases de la luna; las representaciones realizadas coincidieron en alto grado con los dibujos hechos por el docente en la pizarra.

El estudiantado participante pertenecía a cuarto grado, los demás niveles se encontraban en grupos de trabajo realizando prácticas del libro asignadas previamente.

Proceso de aprendizaje del alumnado a partir de las entrevistas realizadas

Una vez ejecutadas las observaciones de las metodologías aplicadas en las clases, se hace necesario contrastar la opinión y percepciones del estudiantado sobre las clases de Ciencias Naturales que reciben, para ello se realizó una corta entrevista estructurada. Previo a la entrevista, se les dio una pequeña explicación sobre las diferentes metodologías de enseñanza con el fin de que pudieran diferenciar entre una estrategia metodológica y otra.

Ante la pregunta: ¿Le gusta la clase que da el maestro?, la mayoría contestó asertivamente y mencionó el maestro sabe usar muy bien la pizarra. Al preguntárseles si tienen gusto por las clases de Ciencias Naturales más de la mitad respondió de forma asertiva.

La mayoría del alumnado entrevistado afirmó que el personal docente utiliza la pizarra como principal instrumento para la explicación de los temas de Ciencias Naturales; pocos mencionaron el uso de prácticas u otros objetos fuera de la clase como complemento al uso de la pizarra. 
Al consultárseles sobre otras formas por medio de las cuales les gustaría aprender de Ciencias Naturales, una gran parte mencionó actividades tales como juegos, uso de más materiales, uso de computadoras y exploración fuera del aula.

Concepto de Ciencias Naturales a través de dibujos realizados por estudiantes de la comunidad indígena Cabécar

Adicional a la entrevista se solicitó a cada estudiante realizar un dibujo para representar lo que entiende por Ciencias Naturales.

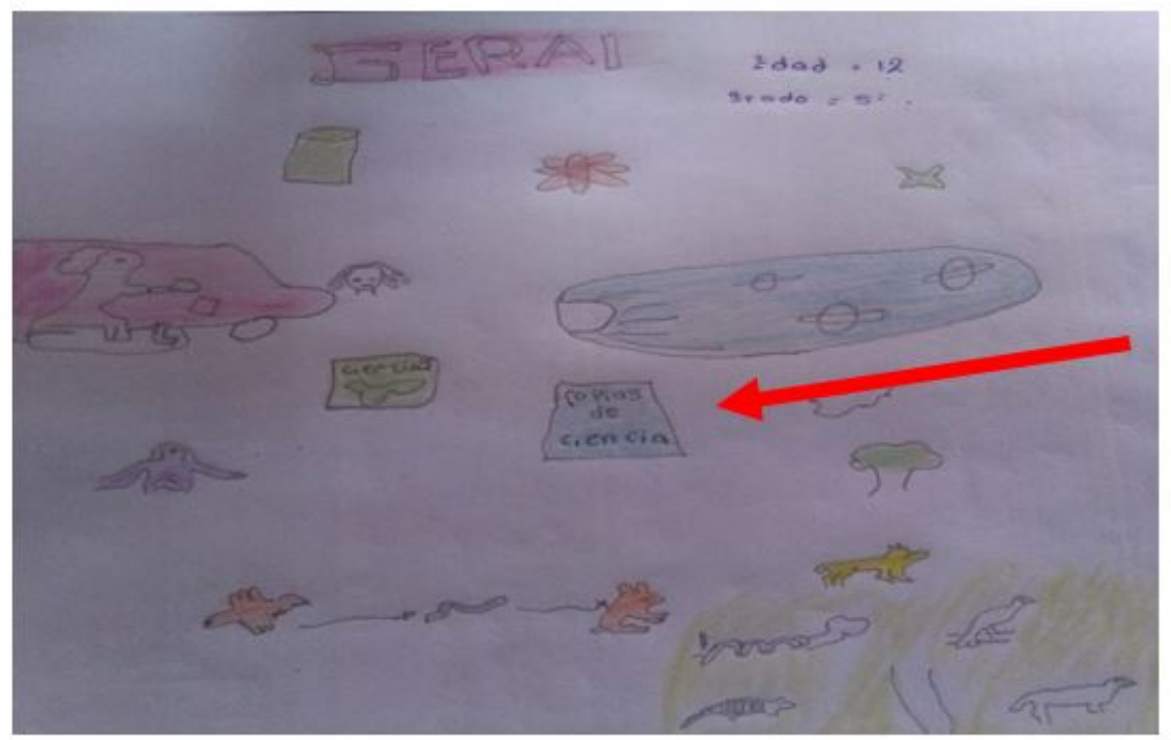

FIGURA 1.

Concepción de las Ciencias Naturales. Estudiante de 12 años

Fuente: Dibujo elaborado por un estudiante de 12 años que cursa el quinto grado en la escuela Gavilán (2012)

En la Figura 1, un estudiante de 12 años de edad y quien cursa el quinto grado en la escuela Gavilán, conceptualiza la ciencia en función de los contenidos temáticos explicados por el docente. Destaca el elemento marcado, denominado copias de ciencias (señalado con la flecha) el cual según lo indicado por el estudiante al no tener la escuela libros para todos, el docente les facilita este recurso para las explicaciones, asimismo se detallan otras representaciones como el sistema solar, la cadena alimenticia, un libro, un carro, un pájaro, un avión, entre otros elementos. 


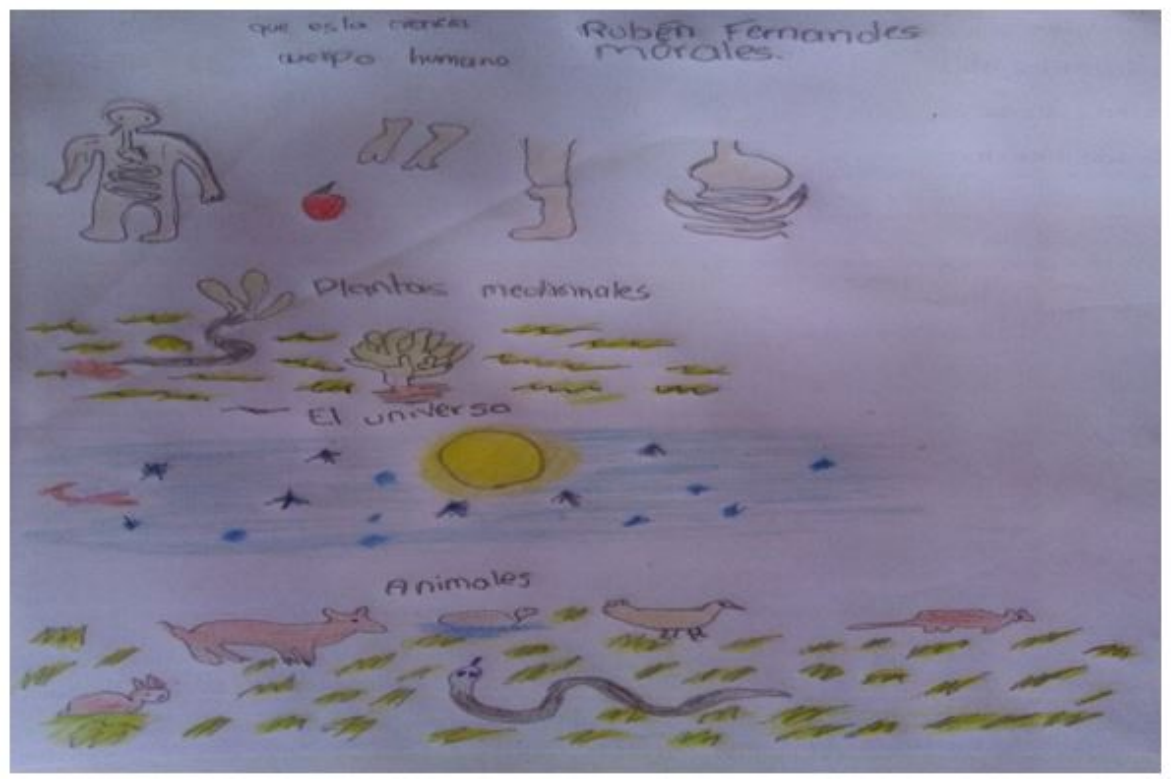

\section{FIGURA 2}

Concepción de las Ciencias Naturales. Estudiante de 8 años

Fuente: Dibujo elaborado por un estudiante de 8 años que cursa el cuarto grado en la escuela Jabuy (2012).

La Figura 2, realizada por un niño de la escuela de Jabuy (la escuela más alejada geográficamente de los demás centros educativos) representa, el cuerpo humano, plantas medicinales, el universo y los animales. No se observó ningún dibujo relacionado con los artefactos tecnológicos.

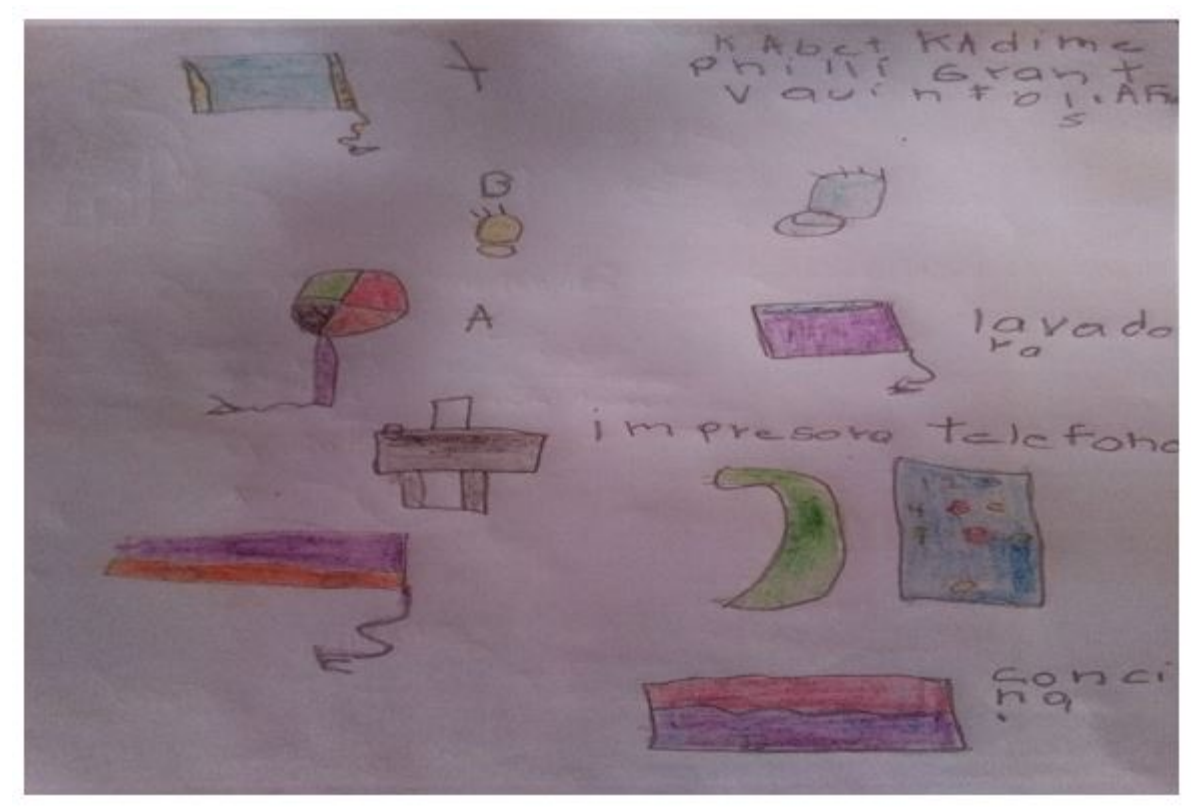

\section{FIGURA 3}

Concepción de las Ciencias Naturales. Estudiante de 9 años

Fuente: Dibujo elaborado por un estudiante de 9 años que cursa el quinto grado en la escuela Vesta (2012).

En la Figura 3 el dibujo realizado por un niño de 9 años quien cursa el quinto grado en la escuela Vesta, se observan objetos tecnológicos (principalmente artefactos eléctricos tales como lavadora, impresora, teléfono, cocina), lo cual responde a los contenidos temáticos explicados por la docente previamente, delimitando así el alcance del aprendizaje en general de las ciencias. 


\section{Estrategias didácticas identificadas}

Las estrategias didácticas utilizadas el personal docente participante se basan en una única estrategia de enseñanza de tipo tradicional-expositiva, en la cual el profesorado asume el papel de protagonista dentro del proceso de enseñanza-aprendizaje.

Lo anterior es notable al analizar que en su mayoría las lecciones se desarrollan a través de clases magistrales impartidas por parte de cada docente utilizando prácticamente una única y tradicional metodología de enseñanza.

En este punto es importante destacar que el uso casi exclusivo de la pizarra como instrumento de enseñanza, limita las posibilidades del alumnado para desarrollar habilidades y destrezas para el aprendizaje de las Ciencias Naturales en un ambiente escolar inclusivo dirigiéndolo hacia un aprendizaje de tipo memorístico de corto plazo.

Las observaciones evidencian la orientación conductista en el desarrollo de las lecciones; basándose en lo indicado por Hernández (1998), respecto a la concepción del personal docente desde el paradigma con enfoque conductista: "para este enfoque, el trabajo del maestro consiste en desarrollar una adecuada serie de arreglos de contingencia de reforzamiento y control de estímulos para enseñar" (p.94).

Las explicaciones magistrales en la pizarra y la utilización de otros materiales como por ejemplo la arcilla, constituyen elementos estimuladores de la enseñanza bajo el control docente; bajo este tipo de enfoque el profesorado se convierte en el principal protagonista de la lección mientras el estudiantado escucha pasivamente lo que este les menciona. Para Bruner (1966, citado por EDUCARCHILE, 2011) debe ser inherente "integrar conocimientos con la acción y sobre todo hacer atractiva la enseñanza" (p.46), por tal motivo es que algunos estudiantes no prestan atención y la monotonía les causa cansancio.

El papel pasivo del alumnado fue el común denominador de las clases observadas, si bien es cierto, se evidenció algún tipo de intercambio de información docente-estudiante, en la mayoría de casos el docente asume el papel protagónico. Las clases se desarrollaron a través de vías de comunicación verticales, lo cual reforzaba la posición del estudiantado como ente receptor; ante esto Rivas y Telleria (2005) afirman: "cuando la enseñanza está centrada en el docente, el estudiante responde preguntas de las que el docente conoce la respuesta y es poco frecuente que los estudiantes hagan preguntas importantes" (p.1).

En relación con las respuestas del alumnado, estos demandan el uso de materiales individuales, principalmente en lo que respecta al libro de texto, también son enfáticos acerca del interés de aprender con el uso de computadoras, dinámicas de juegos, trabajo de campo y otros materiales.

Los tres centros educativos tienen limitaciones de recursos, sin embargo el personal docente puede apegarse a la realidad y aprovechar otro tipo de insumos presentes en la zona, los cuales pueden facilitar el desarrollo de dinámicas de grupo, el trabajo de campo y la relación general con el entorno.

Los dibujos realizados por el estudiantado denotan la poca relación que estos encuentran entre la ciencia y la cotidianidad desde el punto de vista holístico, delimitando estrictamente la ciencia con los contenidos temáticos evaluados por el cuerpo docente, lo cual evidencia su papel protagónico en la enseñanza.

En la Figura 1, al estar expuesto a un aprendizaje dirigido y limitado, el estudiante dibujó lo recordado de las clases, no relacionando los contenidos con los fenómenos cotidianos a su alrededor, incluso dibujó un documento con el nombre copias de ciencias; mientras que el dibujo mostrado en la Figura 3, muestra una coincidencia casi total entre la concepción del estudiante de las ciencias naturales con los artefactos mencionados por el docente, lo cual refuerza el efecto conductista de la estrategia pedagógica.

En sus dibujos otros estudiantes representaron las Ciencias Naturales como el sistema digestivo, el sistema circulatorio, las fases de la luna y las mezclas, entre otros, lo cual sugiere una influencia de los últimos temas vistos en clase.

Las respuestas ratifican el modelo educativo basado en el paradigma conductista, evidenciado en la práctica docente. La Universidad Oberta de Catalunya (2014) destaca las funciones del personal docente dentro de este paradigma, al respecto señala: 
- Diagnosticar las necesidades instruccionales.

- Crear y diseñar condiciones para la instrucción.

- Mantener y conducir la instrucción.

- Manejar las técnicas de evaluación (p.3).

Según las respuestas a las entrevistas a la mayoría de estudiantes les gustaría una forma de enseñanza enmarcada por el dinamismo y a través de la cual su propia participación vaya mucho más allá del simple hecho de sentarse en un pupitre a escuchar a su docente hablando y explicando temas.

No obstante, como ya se mencionó, la estrategia predominante es de tipo expositiva-magistral y la información recabada por las observaciones develó un uso muy limitado de otras técnicas de enseñanza. Por ejemplo, en la escuela de Jabuy se utilizó el barro para que el alumnado ejemplificara las fases de la luna, sin embargo, antes de realizar tal actividad, el docente había explicado el tema en la pizarra y las representaciones realizadas por sus estudiantes fueron casi exactas a las vistas en la pizarra.

Si bien es cierto en la escuela Gavilán, en una clase se utilizó arena, leche en polvo y agua para ejemplificar el tema de mezclas, el docente tenía un conocimiento previo de la visita y llama la atención que el tema de mezclas fue denominado por él mismo como un repaso de contenidos temáticos antes explicados, por lo que existe la posibilidad de que dicha actividad (uso de diferentes materiales) haya respondido más a un factor de impresión para el investigador.

Uno de los objetivos primordiales en la enseñanza de las Ciencias Naturales es lograr la interpretación y el entendimiento por parte del estudiantado de los fenómenos naturales y cotidianos a su alrededor, por lo tanto, se torna sumamente importante un papel crítico por parte del cuerpo docente sobre la realidad con el fin de transmitir ese sentido a sus estudiantes.

Únicamente dos estudiantes (del total de participantes) realizaron un dibujo en el cual incluyeron árboles, algún tipo de vegetación y cultivos además de animales como gallinas y cerdos. Un estudiante hizo una referencia al dios Sibü en alusión al creador de la naturaleza en la realidad Cabécar.

La indagación se convierte en un vehículo para propiciar el pensamiento crítico y reflexivo, al respecto López (2012) afirma:

Los profesores deben proporcionar experiencias que permitan a todos sus alumnos desarrollar estrategias de indagación y solución de problemas de forma experta, en un clima de seguridad que permita perfeccionar el pensamiento complejo. Para ello, es necesario conocer los elementos requeridos para realizar buenas preguntas, y reconocer los diferentes tipos de preguntas que se pueden elaborar. (p.48)

Resulta necesario analizar la influencia ejercida por el perfil sociodemográfico imperante en la zona sobre los procesos de enseñanza-aprendizaje. Al tratarse de una zona con limitaciones en todos los campos, se vuelve difícil proyectar un modelo educativo congruente con los utilizados en otras zonas del país en las cuales el acceso a la información y la infraestructura juegan un papel preponderante en la dinámica educativa.

Lo anterior ubica en una plataforma en la cual, a pesar de las limitantes ya mencionadas, existen en la zona otro tipo de recursos valorativos de los cuales el personal docente puede tomarlos como insumos para los procesos de enseñanza aprendizaje.

En el campo de las Ciencias Naturales el entorno brinda una serie de elementos facilitadores para el aprendizaje, incluso el hecho de que muchos estudiantes realicen labores no remuneradas fuera de clases en actividades agrícolas y pecuarias, le puede representar al docente la oportunidad de desarrollar conceptos relacionando las tareas cotidianas con el aprendizaje de las Ciencias Naturales.

En general, a pesar de que la realidad docente en la zona indígena estudiada se enfrenta a una serie de limitaciones a nivel de recursos; el profesorado debe propiciar los espacios para explotar, la creatividad, las habilidades y las destrezas de sus estudiantes y evitar limitarse al uso exclusivo de estrategias de enseñanza en las cuales el estudiantado asume un papel de receptor pasivo. 
El desarrollo de diversas estrategias didácticas representa una alternativa la cual puede ser aprovechada por el personal docente para fomentar un aprendizaje significativo, por ejemplo la estrategia basada en el juego el cual es la mayor forma de entretenimiento de la niñez y como tal,

representa el periodo de aprendizaje sobre cómo está construido su entorno social, lo cual explica por qué muchos juegos aluden a ello; es decir, a través del juego, los chicos y las chicas ponen de manifiesto las enseñanzas que van adquiriendo. (Alonso-Aguirre et ál., 2013, p.80)

Por lo tanto, la diversidad y el dinamismo de las estrategias didácticas se convierten en el medio a través del cual el estudiantado desarrolla diferentes habilidades de cara a enfrentar los retos de la realidad que le rodea.

La labor pedagógica del personal docente en las zonas indígenas se ve también marcada por el contenido de un currículo educativo diseñado de forma genérica sin contemplar las características propias de su entorno; al respecto Bensasson citado por Baronnet (2013) en referencia a las políticas educativas indígenas en México señala: "La planeación educativa y los programas de formación docente son determinados y controlados por el estado nacional de una manera centralizada, lo que desemboca en una educación burocratizada, corrupta, antidemocrática y de baja calidad" (p.191).

De acuerdo con lo anterior a nivel de política educativa resalta la necesidad de elaborar currículos académicos adaptables a los menesteres y las características del entorno indígena los cuales coadyuven a la labor dentro de las aulas.

\section{Conclusiones}

En relación con las estrategias didácticas utilizadas por el personal docente participante, se puede concluir que se ejecuta una práctica de enseñanza de tipo tradicional-expositiva encerrado en un paradigma con enfoque conductista.

Esta influencia de la corriente conductista se evidencia en las acciones realizadas por el estudiantado dentro del aula, el cual respondió siempre a los lineamientos e instrucciones dictadas por cada docente, lo que deja entrever un accionar taxativo del proceso de enseñanza.

A pesar de que algunas de las observaciones reflejaron el uso de técnicas más dinámicas tales como el uso de barro para elaborar las fases de la luna y el uso de diferentes materiales; cada docente mantuvo su rol protagonista y siempre dictaminó las reglas a seguir dentro del proceso educativo.

La principal (y casi única) estrategia didáctica fue la clase magistral basada en explicaciones en la pizarra y el uso de libros de texto.

Las pocas evidencias de variantes en la dinámica educativa observada, siempre conservaron una línea de tipo instruccional, a través de la cual, cada docente tuvo el control del proceso, limitando la criticidad de sus estudiantes.

Las clases de Ciencias Naturales observadas carecen de metodologías de trabajo mediante las cuales el alumnado pueda aprender haciendo. El desarrollo de actividades fuera del aula (trabajo de campo) representaría una forma sencilla para liberar al estudiantado de la monotonía representada por la impartición continua de clases magistrales.

Los estudiantes demandan clases de ciencias más activas, a través de estrategias de enseñanza más dinámicas, las cuales limiten el uso de la pizarra como único instrumento de enseñanza.

No cabe duda que la ausencia de recursos es un factor determinante que condiciona el aprendizaje, dado que la mayoría de estudiantes no tiene ni siquiera un libro para uso individual.

El hecho de que relacionen el aprendizaje de las Ciencias Naturales con los últimos contenidos impartidos en la clase y no con los hechos y fenómenos que les rodean, es reflejo de que el personal docentes, a través de su práctica pedagógica, no ha establecido las bases para conceptualizar el aprendizaje de las Ciencias Naturales en relación con la vida cotidiana y tampoco se ha apropiado de su entorno para potencializar dicha enseñanza. 
Los planeamientos educativos y la elaboración del currículo académico por parte del Ministerio de Educación Pública deben adaptarse a los entornos indígenas, con el fin de colaborar en la búsqueda de metodologías de enseñanza afines a las características del personal docente y los recursos disponibles en el medio.

\section{REFERENCIAS}

Alonso-Aguirre, M. G., Ayala-Reyes, S., Berumen-Campos, G., Cabrera-Morales, N., Castillo-Silva, D. A., GallardoGutiérrez, A. L., y otros. (2013). Desarrollo curricular intercultural de la asignatura de lengua y cultura indigena para la educación secundaria en México. México, D.F.: Coordinación General de Educación Intercultural Bilingüe, Secretaría de Educación Pública

Alonso-Aguirre, M. G. y Gallardo-Gutiérrez, A. L. (2014). Guia para la formación docente de la asignatura estatal de lengua y cultura indigena para la educación secundaria. México, D.F.: Coordinación General de Educación Intercultural Bilingüe, Secretaría de Educación Pública

Baronnet, B. (2013). Lenguas y participación comunitaria en la educación indígena en México AIBR. Revista de Antropología Iberoamericana, 8 (2), 183-208.

Bastiani-Gómez, J., Ruiz-Montoya, L., Estrada-Lugo, E., Cruz-Salazar, T. y Aparicio-Quintanilla, J.A. (2012). Política educativa indígena: práctica docente, castellanización, burocracia y centralización de la educación como limitaciones del éxito pedagógico en la región Ch'ol, Chiapas. Revista Perfiles Educativos, 34 (35), 8-25.

Cuevas, M. (2013). Los grandes inventos y descubrimiento. Recuperado de https://bit.ly/2HcZain

EDUCARCHILE. (2011). Teorías cognitivas: Bruner y Ausubel. Recuperado de https://bit.ly/2SX25wX

Estado de la Nación (2012). Reconocimiento y exigibilidad de los derechos de los pueblos indígenas en Costa Rica: una aproximación. En: Estado de la Nación. Recuperado de https://bit.ly/2FYglTb

Gordillo, M. y Osorio, M. (2003). Educar para participar en ciencia y tecnología. Un proyecto para la difusión de la cultura científica. Revista Iberoamericana de Educación, 32, 165-170

Hernández, G. (1998). Paradigmas en psicología de la educación. México: Paidós.

Hernández, R. Fernández, C. y Baptista, P. (2010). Metodología de la investigación. México: McGraw- Hill

INEC. (2011). CENSO 2011: Población total porpoblación indígena, pertenencia a algún pueblo y población no indigena, según provincia, zona y sexo. Recuperado de https://bit.ly/2nnujCN

Liguori, L. y Noste, M. (2007). Didáctica de las Ciencias Naturales. Argentina: Homo Sapiens.

López, G. (2012). Pensamiento critico en el aula. Recuperado de https://bit.ly/2C6eY2O

Merino, G. (1998). El saber científico escolar: un objeto de conocimiento complejo. Revista Zona Educativa, 12, 56-76

Morin, E. (2004) ¿Qué es el pensamiento complejo? Recuperado de https://bit.ly/1q4EAjT

Morrows, A y Torres, C. (2002) Las teorias de la reproducción social y cultural: Manual critico. Madrid: Popular.

Nicoletti, J. (2003). Nuevas cuestiones éticas. Buenos Aires: Prometeo-UNLaM

Organización Internacional del Trabajo (OIT). (1989). Convenio OIT $N^{\circ} 169$. Sobre pueblos indígenas y tribales en paises independientes. Recuperado de https://bit.ly/2TU42ui

Ontoria, A. (1999). Los mapas conceptuales en el aula. España: Narce

Rodríguez, G. (2009). Un nuevo enfoque para la educación indígena bribri y cabécar. Recuperado de https://bit.ly/2 HE7Bn8

Rivas, M. y Telleria, M. (2005). La lectura como estrategia de enseñanza de las Ciencias Naturales y matemática. Recuperado de https://bit.ly/2AMUDgJ

Sanmartí, N. (2002). Didáctica de las ciencias en la educación cientifica obligatoria. Madrid: Síntesis Educación

Solano, E. (2000). La población indigena de Costa Rica según el Censo 2000. Recuperado de https://bit.ly/2RMoEau

Universidad de Costa Rica. (2016). Atlas de desarrollo humano cantonal de Costa Rica 2016. Recuperado de https:/ /bit.ly/2SS10qa 
Cynthia Vílchez-Durán. Metodología para la enseñanza de las Ciencias Naturales empleada por docent...

Universidad Oberta de Catalunya. (2014). Paradigmas en educación, apuntes de Psicología del Desarrollo. Recuperado de https://bit.ly/2FuApMY

Us, P. (2009). La práctica de la interculturalidad en el aula. Costa Rica: Coordinación Educativa y Cultural Centroamericana

Valladares, L. (2010). La educación científica intercultural y el enfoque de las capacidades. Revista CTS, 6, 39-69.

Villegas, J. (30 octubre, 2012). Nueve liceos no lograron que un solo alumno ganara bachillerato. En: La Nación. Recuperado de https://bit.ly/2HfnjF7

Winch, P. (1987). Comprender una sociedad primitiva. Barcelona: Paidós Ibérica.

\section{BY-NC-ND}

